\title{
Impact of a multidisciplinary care bundle for necrotizing skin and soft tissue infections: a retrospective cohort study
}

Tomas Urbina', Camille Hua ${ }^{2,3}$, Emilie Sbidian ${ }^{2,3,4}$, Romain Bosc ${ }^{5}$, Françoise Tomberli ${ }^{6}$, Raphael Lepeule ${ }^{7,8}$, Jean-Winoc Decousser ${ }^{3,8,9}$, Armand Mekontso Dessap ${ }^{1,3,10}$, Olivier Chosidow ${ }^{2,3,9}$ and Nicolas de Prost ${ }^{1,3,10^{*}}$ (D) on behalf of the Henri Mondor Hospital Necrotizing Fasciitis group

\begin{abstract}
Background: Necrotizing skin and soft tissue infections (NSTIs) require both prompt medical and surgical treatment. The coordination of multiple urgent interventions by care bundles has improved outcome in other settings. This study aimed to assess the impact of a multidisciplinary care bundle on management and outcome of patients with NSTIs.

Methods: Patients with NSTIs admitted between 2006 and 2017 were compared according to admission before or after bundle implementation (2012-2013). This bundle consisted mainly in (1) the creation of a multidisciplinary task force; (2) management guidelines on empirical antibiotics, intensive care unit admission criteria, a triage algorithm to accelerate operating room access; and (3) an active communication policy. Patient recruitment and management were compared between pre- and post-implementation periods. Main outcome was day 60-censored hospital survival.

Results: Overall, 224 patients were admitted: 60 before, 35 during, and 129 after bundle implementation. Admission after implementation was associated with increased yearly admissions (10 [8-13] vs 30 [24-43] patients/year, $p=0.014$ ) and decreased mortality (30 vs $15 \%, \mathrm{HR}=0.49[0.26-0.92] ; p=0.026$ ) but was no longer a protective factor for mortality after adjustment on confounding factors (adjusted $\mathrm{HR}=0.90[0.43-1.88], p=0.780$ ). There was no significant difference regarding time to surgery (0 [0-1] vs $0[0-1]$ days, $p=0.192)$ or rate of antibiotic treatment within $24 \mathrm{~h}$ ( $98 \%$ vs $99 \%, p>0.99$ ).
\end{abstract}

Conclusions: Implementation of a multidisciplinary care bundle for NSTIs was feasible, but in a retrospective study from an already experienced center was not associated with significantly increased survival after adjustment.

Keywords: Necrotizing skin and soft tissue infections, Necrotizing fasciitis, Patient care bundles, Multidisciplinary management, Time to debridement, Mortality

\section{Background}

Necrotizing skin and soft tissue infections (NSTIs) are rare, life-threatening bacterial infections resulting in extensive tissue necrosis and destruction. Hospital mortality ranges from 9.3 to $29.3 \%$, with disabling sequelae for $30 \%$ of survivors [1-3]. With an incidence of

\footnotetext{
*Correspondence: nicolas.de-prost@aphp.fr

1 Service de Réanimation Médicale, Hôpitaux Universitaires Henri Mondor, Assistance Publique-Hôpitaux de Paris (AP-HP), Créteil, France

Full list of author information is available at the end of the article
}

$4 / 100,000$ persons per year [4], initial misdiagnosis is frequent [5], leading to a delayed surgical debridement of infected tissues, one of the main modifiable prognostic factors [6]. Even when diagnostic issues have been overcome, further delays can occur because of logistical and technical difficulties regarding operating room emergency access [7]. Management in high case volume centers has been associated with enhanced survival [8], while a recent Cochrane review highlighted the lack of high quality evidence for a benefit on mortality for any single intervention [9]. This implies the need for a multimodal 
approach of NSTIs, as coordination of multiple urgent interventions has led to improved outcomes in other settings [10-13]. We hypothesized that standardizing and organizing early management at the hospital level would further improve patient care in our tertiary center, and implemented a multidisciplinary care bundle for patients with NSTIs. The current study aims at assessing the impact of this dedicated multimodal care protocol on the following endpoints: (1) yearly patient recruitment; (2) hospital mortality and secondary outcomes; and (3) early patient management, assessed with pre-defined endpoints.

\section{Methods}

\section{Patients}

We conducted a retrospective cohort study including all consecutive adult patients ( $\geq 18$ years) admitted to our hospital for surgically confirmed NSTI from January 1st 2006 to December 31st 2017. Patients admitted between 2006 and 2013 were identified using the French national hospital database (Program for Medicalization of Information Systems) with the International Classification of Diseases diagnostic codes for "gangrene", "infectious myositis", "necrotizing fasciitis" and "cellulitis". Patients admitted after 2014 were identified from a prospective NSTI database. Patients were excluded if NSTI was not surgically confirmed. Macroscopic appearance of tissues during operation (i.e., swollen, dull gray with a thin, brownish exudate with or without necrosis) was used as the main inclusion criterion as it is the gold standard for diagnosis of NSTI in the most recent guidelines [14] and no change within the surgical team occurred throughout the study period. Patients were also excluded if upon medical chart review NSTI was not the main admission diagnosis, or if there were missing data regarding the main outcome or two or more of the key management endpoints (i.e., time to surgery, time to antibiotics, adequacy of antibiotics to guidelines, admission to intensive care). Follow-up was conducted by review of electronic medical charts until end of study inclusion (December 31st 2017), using last available information (outpatient consultation or hospital discharge) to estimate follow-up time.

Patients received information during hospital stay that data abstracted from their medical charts could be used for research purposes. Data were anonymized and compiled according to the requirements of the Commission Nationale Informatique et Liberté (registration number 2003722) and the study was approved by the Comite de Protection des Personnes Ile-de-France V on March 8th 2018 (reference \# 16165). The study has been reported according to the STROBE guidelines regarding observational cohort studies.

\section{NSTI care bundle}

Through 2012 to 2013, a multidisciplinary bundle of care for NSTIs was progressively implemented in our tertiary referral center. It consisted in (1) the creation of a multidisciplinary task force involving intensive care physicians, dermatologists, surgeons, infectious diseases practitioners, microbiologists, and radiologists; (2) the use of a triage algorithm including a 24/7 on-call dermatologist for patient referral and a multidisciplinary bedside assessment to facilitate access to the operating room; (3) the implementation of local management guidelines addressing empiric antibiotic treatment, intensive care unit (ICU) admission criteria, prioritization for operating room access, adequate specimen collection for laboratory detection of responsible microorganisms, systematic "second-look" surgery recommendation $24 \mathrm{~h}$ after initial surgical debridement, together with a routine multidisciplinary bedside reassessment during the post-operative period; (4) the prospective identification of all NSTI cases admitted to our institution as well as their inclusion in a dedicated database; (5) trimesterly review of all NSTI cases by the multidisciplinary task force; and (6) the conduction of research projects and an active communication policy towards the medical community about the existing bundle. The main elements of this bundle are presented in Additional file 1: Figures S1 and S2 and Additional file 2: Appendix S1.

\section{Study design}

Using a before-after design, we compared patients from the pre- and post-implementation period (2006-2011 vs 2014-2017) for the following variables: number of yearly admissions, patients' clinical characteristics, key predefined early management endpoints (i.e., time from hospital admission to first surgical debridement (measured in days), antibiotic administration within $24 \mathrm{~h}$ of hospital admission, adequacy of antibiotics to guidelines, ICU admission), number of surgical debridements, length of hospital stay and hospital mortality. Shock was defined as need for vasopressors, amputation was defined as amputation of at least a limb segment, of external genitalia or of perineal sphincters. Initial symptoms and their time of onset were recovered from medical charts or considered as missing if not reported. Microbiological data were obtained from samples collected during the first surgery, blood cultures, subcutaneous and bullae punctures collected before or on the day of the first surgery. Samples obtained from subsequent surgical procedures were not included. Results from all samples were merged to categorize infections as mono- or polymicrobial for each patient. All data were collected upon medical chart review. Due to the progressive implementation of the different bundle items, patients admitted between January 
2012 and December 2013, the defined implementation period, were excluded from the final analysis. The primary endpoint was 60-day-censored hospital survival. Primary outcome and key management outcomes had been defined a priori. The adequacy of empirical antibiotic treatment was defined according to the most recent French [15] and international guidelines [1-3].

\section{Statistical analysis}

Continuous variables were reported as median [1st3rd quartiles] and categorical data as percentages. No imputation was performed for missing data, except for comorbidities, imputed as absent if not otherwise stated. Differences between patients included during the preand post-implementation periods were tested using the Mann-Whitney non-parametric test for continuous variables, and the Fisher's exact test or the Chi-squared test for categorical variables, according to sample size. A sensitivity analysis for the impact of bundle implementation on pre-defined management endpoints was conducted including only patients presenting with shock. Factors associated with day 60-censored hospital survival were identified using uni- and multivariable Cox proportional hazards regression models, hazard ratios (HR) and their $95 \%$ confidence interval $(\mathrm{CI})$ were computed. Features yielding a $p$ value $<0.05$ in univariable analysis were included in the multivariable model, with a manual backwards stepwise elimination procedure of variables displaying a $p$ value greater than 0.10 until reaching the final model. No imputation was made and patients with missing data for one of the variables from the model were excluded from the analysis. Sensitivity analyses were conducted applying the same model to either the whole study population, including patients from the implementation period, or to only patients from the pre- and post-implementation periods presenting with shock. Survival curves were drawn using the Kaplan-Meier survival analysis, and the log rank test was used to compare the differences. Significance was defined as two-sided $p$ value $<0.05$. Data were collected and entered into a Microsoft Excel 2010 (Microsoft Corporation, Redmond, WA) spreadsheet. All statistical analyses were performed using the R software (v 2.12.0; http://cran.r-project.org). Tables and figures were made using $\mathrm{R}$ and GraphPad Prism 5 (GraphPad Software Inc, La Jolla, Calif).

\section{Results}

During the study period (2006-2017), 282 patients were admitted with a diagnosis of NSTI, including 44 patients for whom surgically confirmed NSTI was not the main admission diagnosis (no surgical confirmation, $n=37$, and hospital admission for another reason than NSTI care, $n=7$ ) and 14 patients with missing data [regarding main outcome $(n=10)$, regarding two or more predefined key management endpoints $(n=4)]$, leaving 224 patients available for study inclusion. Of these, 35 patients were admitted during the implementation period, resulting in 189 patients available for analysis (60 from the pre-implementation period and 129 from the post-implementation period) (see Additional file 1: Figure S3). The median follow-up time was 86 [30-367] days.

\section{Impact of the NSTI care bundle on patient recruitment and characteristics}

Between the pre- and post-implementation periods, there was a marked increase in the yearly number of patients admitted to our center for NSTIs $(10$ [8-13] vs 30 [2443] patients/year, $p=0.014$; Fig. 1 ), with no increase in the proportion of patients transferred from another center $(52 \%$ vs $53 \%, p=0.939)$. Regarding patients' characteristics upon admission, the only significant difference was a lower rate of nosocomial NSTIs during the post-implementation period ( $33 \%$ vs $13 \%, p=0.002$ ) and a trend for NSTIs to more frequently affect the lower limbs $(67 \%$ vs $80 \%, p=0.060)$. There were no significant differences between the two periods regarding non-steroidal anti-inflammatory drug and antibiotic use before hospital admission, the presence of shock upon admission (Table 1), or the microbiological documentation of NSTIs (see Additional file 1: Table S1).

\section{Impact of the NSTI care bundle on day 60-censored hospital survival and secondary outcomes}

Overall, 51 patients (23\%) had died 60 days after admission, with a significantly lower mortality after bundle implementation $(30 \%(n=18 / 60)$ vs $15 \%(n=20 / 129)$, $p=0.034$ ). Length of hospital stay was shorter (29 [17$41]$ vs 21 [11-34] days, $p=0.041)$, with no difference in the rate of amputation $(22 \%$ vs $16 \%, p=0.418)$ or the

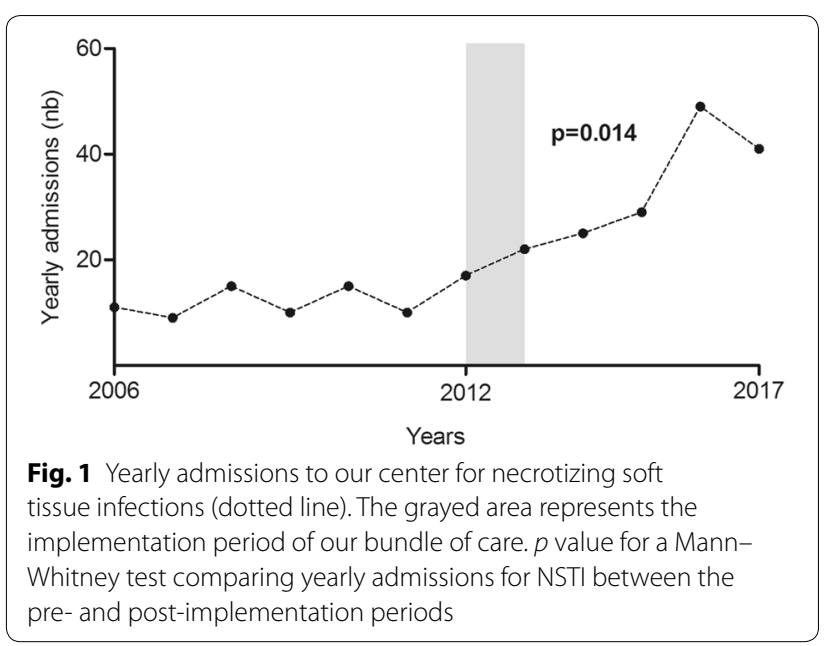


Table 1 Demographics, comorbidities and clinical features upon admission of patients with necrotizing skin and soft tissue infections before $(n=60)$, during $(n=35)$ and after $(n=129)$ the implementation of a dedicated multimodal and multidisciplinary bundle

\begin{tabular}{|c|c|c|c|c|c|c|}
\hline & Available data & $\begin{array}{l}\text { All patients } \\
n=224\end{array}$ & $\begin{array}{l}\text { Pre-implementation } \\
\text { period (2006-2011) } \\
n=60\end{array}$ & $\begin{array}{l}\text { Implementation } \\
\text { period (2012- } \\
2013) \\
n=35\end{array}$ & $\begin{array}{l}\text { Post- } \\
\text { implementation } \\
\text { period (2014-2017) } \\
n=129\end{array}$ & $p^{a}$ \\
\hline \multicolumn{7}{|l|}{ Demographics } \\
\hline Age, years, median (IQR) & 224 & $64(53-74)$ & $66[55-76]$ & $68[60-75]$ & $61[53-72]$ & 0.193 \\
\hline Male gender, $n(\%)$ & & $127(56.7)$ & $34(56.7)$ & $17(48.6)$ & $76(58.9)$ & 0.894 \\
\hline \multicolumn{7}{|l|}{ Comorbidities, $n(\%)$} \\
\hline Diabetes mellitus & 224 & $83(37.1)$ & $18(30.0)$ & $12(34.3)$ & $53(41.1)$ & 0.192 \\
\hline Immunodeficiency & & $58(25.9)$ & $13(21.7)$ & $14(40.0)$ & $31(24.0)$ & 0.863 \\
\hline HIV infection & & $2(0.9)$ & $1(1.7)$ & $1(2.9)$ & $0(0.0)$ & 0.694 \\
\hline Cancer & & $21(9.4)$ & $5(8.3)$ & $7(20.0)$ & $9(7.0)$ & 0.974 \\
\hline Corticosteroids & & $36(16.1)$ & $6(10.0)$ & $9(25.7)$ & $21(16.3)$ & 0.355 \\
\hline Obliterating arteritis of the lower limbs & & $24(10.7)$ & $6(10.0)$ & $4(11.4)$ & $14(10.9)$ & $>0.99$ \\
\hline Liver cirrhosis & & $9(4.0)$ & $1(1.7)$ & $2(5.7)$ & $6(4.7)$ & 0.550 \\
\hline Chronic kidney disease & & $25(11.2)$ & $7(11.7)$ & $5(14.3)$ & $13(10.1)$ & 0.939 \\
\hline Chronic alcohol consumption & & $27(12.1)$ & $7(11.7)$ & $5(14.3)$ & $15(11.6)$ & $>0.99$ \\
\hline Obesity & & $57(25.4)$ & $16(26.7)$ & $10(28.6)$ & $31(24.0)$ & 0.834 \\
\hline \multicolumn{7}{|l|}{ Prior to admission } \\
\hline $\begin{array}{l}\text { Time from first symptom, days, median } \\
\text { (IQR) }\end{array}$ & 214 & $5[2-10]$ & $6[2-12]$ & $4[1-15]$ & 5 [3-9] & 0.848 \\
\hline Antibiotic treatment, $n(\%)$ & 221 & $137(61.2)$ & $33(55.9)$ & $20(58.8)$ & $84(65.6)$ & 0.267 \\
\hline NSAID use, $n(\%)$ & 222 & $46(20.5)$ & $9(15.0)$ & $4(11.8)$ & $33(26.0)$ & 0.143 \\
\hline Transferred from another center, $n(\%)$ & 223 & $116(51.8)$ & $31(51.7)$ & $16(47.1)$ & $69(53.5)$ & 0.939 \\
\hline \multicolumn{7}{|l|}{ Presentation upon admission } \\
\hline Nosocomial infection, $n(\%)$ & 222 & $45(20.1)$ & $20(33.3)$ & $8(23.5)$ & $17(13.4)$ & 0.002 \\
\hline Infection site, $n(\%)$ & 223 & & & & & \\
\hline Inferior limbs & & $173(77.2)$ & $40(66.7)$ & $30(85.7)$ & $103(80.5)$ & 0.060 \\
\hline Superior limbs & & $19(8.5)$ & $8(13.3)$ & $1(2.9)$ & $10(7.8)$ & 0.351 \\
\hline Abdomino-perineal infection & & $38(17.0)$ & $13(21.7)$ & $4(11.4)$ & $21(16.4)$ & 0.503 \\
\hline Cervico-facial infection & & $2(0.9)$ & $0(0.0)$ & $0(0.0)$ & $2(1.6)$ & 0.833 \\
\hline Other & & $1(0.4)$ & $0(0.0)$ & $0(0.0)$ & $1(0.8)$ & 1 \\
\hline Multifocal infection, $n(\%)$ & 223 & $23(10.3)$ & $5(8.3)$ & $4(11.4)$ & $14(10.9)$ & 0.770 \\
\hline Shock, $n(\%)$ & 220 & $91(40.6)$ & $30(50.8)$ & $12(34.3)$ & 49 (38.9) & 0.170 \\
\hline
\end{tabular}

HIV human immunodeficiency virus, NSAID non-steroidal anti-inflammatory drug

${ }^{a} p$ values for univariate comparison of the pre- and post-implementation periods; Chi-squared test or Fisher's exact test were used for categorical data according to sample size, Mann-Whitney's test were used for continuous variables due to non-parametrical distribution

median number of debridements $(1[1-2]$ vs 1 [1-2], $p=0.587$ ). Consistently, admission during the postimplementation period was a protective factor for day 60 -censored hospital survival in univariable analysis $(\mathrm{HR}=0.49$ [0.26-0.92]; $p=0.026)$ (Fig. 2). All admission characteristics associated with mortality in univariable analysis are presented in Table 2. In a multivariable Cox model, the variables remaining associated with mortality were age as a continuous variable (adjusted (a) $\mathrm{HR}=1.04$ [1.01-1.07], $p=0.011$ ), immunodeficiency ( $\mathrm{aHR}=2.20$ [1.09-4.44], $p=0.028)$, the nosocomial status of the infection $(\mathrm{aHR}=2.28[1.00-5.16], p=0.049)$ and the presence of shock $(\mathrm{aHR}=8.13[3.26-20.20], p<0.001)$. Antibiotics administered before admission were a protective factor $(\mathrm{aHR}=0.36[0.17-0.75], p=0.006)$. The admission period was no longer associated with day 60 -censored hospital survival $(\mathrm{aHR}=0.90$ [0.43-1.88], $p=0.780$ ) (Table 2). Two sensitivity analyses including either all cohort patients, even those from the implementation period (2011-2012), or only those presenting with shock upon admission, yielded consistent results (see Additional file 1: Tables S2 and S3). The main management endpoints identified a priori (time to first surgery, rate of patients undergoing surgery in the first $24 \mathrm{~h}$, rate 


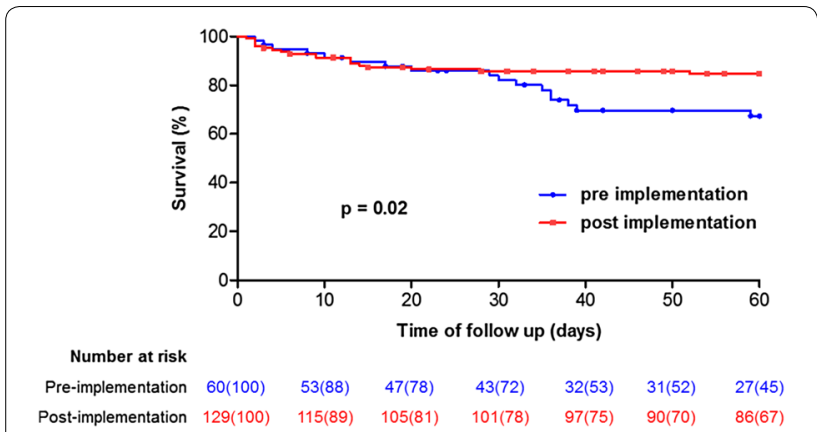

Fig. 2 Kaplan-Meier survival curves comparing patients admitted during the pre- (blue line) and post- (red line) bundle of care implementation periods. $p$ value comes from an unadjusted log rank test. Survival was censored at 60 days

of patients receiving antibiotics in the first $24 \mathrm{~h}$ and rate of adequacy of first antibiotherapy to guidelines) were not significantly associated with survival (see Additional file 1: Table S4).

\section{Impact of the NSTI care bundle on patient management}

No significant differences in key pre-defined management endpoints were noted between the pre- and postimplementation periods (Fig. 3). The time to first surgery was $0[0-1]$ vs $0[0-1]$ days $(p=0.192)$, with $78 \%$ of patients undergoing surgery within the first $24 \mathrm{~h}$ in both groups. Antibiotics were administered within the first $24 \mathrm{~h}$ in more than $98 \%$ of patients in both groups, and in accordance to guidelines in more than $90 \%$ of cases. The rate of ICU admission did not significantly differ between the two periods, nor did the rate of ICU admission within the first $24 \mathrm{~h}$ (see Additional file 1: Table S5). A sensitivity analysis including the most severe patients, i.e., those presenting with shock, did not reveal any significant differences for these same endpoints (see Additional file 1: Table S6). Of 224 patients, seven received intravenous immunoglobulins: one from the implementation period and six from the post-implementation period. Hyperbaric oxygen therapy was not used.

\section{Discussion}

The main results of this retrospective study assessing the impact of the implementation of a multidisciplinary NSTI care bundle are as follows: (1) the NSTI care bundle was associated with a marked increase in yearly admissions; (2) it was associated with an increase in day 60-censored hospital survival in univariable analysis, which was not maintained after adjustment for admission characteristics; and (3) it did not allow for significantly altering key pre-defined patient management endpoints.
The increase in yearly patient recruitment (Fig. 1) per se could be an interesting benefit of our bundle. Indeed, in a recent study by Audureau et al. [8], patients managed in centers admitting a high volume of NSTI cases (defined by 3 or more yearly cases) had a lower 28-day mortality, even after adjusting for potential confounding factors. Bernal et al. found that mortality was lower for patients whose initial surgical debridement was performed by a surgeon experienced in NSTIs [16], further highlighting the potential benefits of being managed in centers with high case volumes. Although yearly patient recruitment increased between the pre- and post-implementation periods, there was no increase in the rate of patients who were transferred to our center from another facility. As the incidence of NSTI is not thought to be rising $[17,18]$, this could nevertheless be due to our communication policy, by an increase of direct referral by pre-hospital medical teams or general practitioners as well as by an increase of spontaneous patient consultation to our center's "dermatological emergency ward". As the inclusion criteria for our study was surgically confirmed NSTI, an increase in incidence due to erroneous diagnosis is unlikely, but could be due to the increased awareness, experience and training of physicians regarding NSTI in our center.

The survival benefit associated with the post-implementation period was not maintained after adjustment for admission characteristics (Fig. 2, Table 2). The main hypothesis is that bundle impact could have been undermined because our center was already experienced in the management of NSTIs. Indeed, there was no significant difference regarding the main management endpoints between the pre- and post-implementation periods (Fig. 3). The rate of patients rapidly undergoing surgery and receiving adequate antibiotics, even before implementation of the bundle, is difficult to compare to the literature. It was markedly high compared to some studies [19], and lower compared to others [20, 21]. Nevertheless, the rate of patients transferred from other centers in our series, of more than $50 \%$ both in the pre- and postimplementation periods, is much higher than in previous studies evaluating NSTIs at a national level (Audureau et al. 13\% [8], Holena et al. 10\% [22], Ingraham et al. 30\% [23]) but similar to that of series from experienced centers such as the one by Bernal et al. (59\%) [16]. Besides this homogenous management, other explaining factors could be a lack of power due to the rarity of NSTIs or a change in patient characteristics associated with the increase in the number of admissions, as highlighted by the lesser proportion of patients with nosocomial infections, known to be more severe [24, 25]. Figure 1 illustrates that the mortality difference between groups seems to develop after day 30 . We speculate this could reflect 
Table 2 Admission characteristics associated with hospital mortality censored at day 60 amongst patients admitted in the pre- and post-implementation periods

\begin{tabular}{|c|c|c|c|c|c|c|c|}
\hline & $\begin{array}{l}\text { Available } \\
\text { data }\end{array}$ & $\begin{array}{l}\text { Survivors } \\
n=151\end{array}$ & $\begin{array}{l}\text { Non-survivors } \\
n=38\end{array}$ & $\begin{array}{l}\text { Unadjusted } \\
\text { hazard ratio } \\
{[95 \% \mathrm{Cl}]^{\mathrm{a}}}\end{array}$ & $p^{\mathbf{b}}$ & $\begin{array}{l}\text { Adjusted } \\
\text { hazard ratio } \\
{[95 \% \mathrm{Cl}]^{c}}\end{array}$ & $p^{d}$ \\
\hline \multicolumn{8}{|l|}{ Inclusion period, $n(\%)$} \\
\hline Post-implementation & 129 & $109(72.2)$ & $20(52.6)$ & $0.49[0.26-0.92]$ & 0.026 & $0.90[0.43-1.88]$ & 0.780 \\
\hline Pre-implementation & 60 & $42(27.8)$ & $18(47.4)$ & - & - & & \\
\hline \multicolumn{8}{|l|}{ Demographical data } \\
\hline Age, years, median (IQR) & 189 & $61[51-72]$ & 71 [59-80] & $1.00[1.00-1.10]$ & 0.018 & $1.04[1.01-1.07]$ & 0.011 \\
\hline Male gender, $n(\%)$ & & $86(57.0)$ & $24(63.2)$ & - & 0.510 & & \\
\hline \multicolumn{8}{|l|}{ Comorbidities, $n$ (\%) } \\
\hline Diabetes mellitus & 189 & $58(38.4)$ & $13(34.2)$ & - & 0.520 & & \\
\hline Immunodeficiency & & $26(17.2)$ & $18(47.4)$ & $3.30[1.70-6.20]$ & $<0.001$ & $2.20[1.09-4.44]$ & 0.028 \\
\hline HIV infection & & $1(0.7)$ & $0(0.0)$ & - & $>0.99$ & & \\
\hline Cancer & & $9(6.0)$ & $5(13.2)$ & - & 0.140 & & \\
\hline Corticosteroids & & $16(10.6)$ & $11(28.9)$ & $2.70[1.40-5.50]$ & 0.005 & & \\
\hline Obliterating arteritis of the lower limbs & & $15(9.9)$ & $5(13.2)$ & - & 0.580 & & \\
\hline Liver cirrhosis & & $4(2.6)$ & $3(7.9)$ & - & 0.100 & & \\
\hline Chronic kidney disease & & $10(6.6)$ & $10(26.3)$ & $3.50[1.70-7.20]$ & $<0.001$ & - & - \\
\hline Chronic alcohol consumption & & $20(13.2)$ & $2(5.3)$ & - & 0.190 & & \\
\hline Obesity & & $42(27.8)$ & $5(13.2)$ & - & 0.061 & & \\
\hline \multicolumn{8}{|l|}{ Prior to admission } \\
\hline Time from first symptom, days, median (IQR) & 180 & $5[3-9]$ & $3[1-8]$ & - & 0.410 & & \\
\hline Antibiotic treatment, $n(\%)$ & 187 & 99 (65.6) & $18(47.4)$ & $0.51[0.27-0.97]$ & 0.041 & $0.36[0.17-0.75]$ & 0.006 \\
\hline NSAID use, $n(\%)$ & 188 & $38(25.2)$ & $4(10.5)$ & - & 0.076 & & \\
\hline Transferred from another center, $n(\%)$ & 189 & $79(52.3)$ & $21(55.3)$ & - & 0.670 & & \\
\hline \multicolumn{8}{|l|}{ Presentation upon admission } \\
\hline Nosocomial infection, $n(\%)$ & 188 & $24(15.9)$ & $13(34.2)$ & $2.50[1.30-5.00]$ & 0.007 & $2.28[1.00-5.16]$ & 0.049 \\
\hline Infection site, $n(\%)$ & 188 & & & & & & \\
\hline Inferior limbs & & $114(75.5)$ & $29(76.3)$ & - & 0.600 & & \\
\hline Superior limbs & & $15(9.9)$ & $3(7.9)$ & - & 0.690 & & \\
\hline Abdomino-perineal & & $27(17.9)$ & $7(18.4)$ & - & 0.960 & & \\
\hline Cervico-facial & & $2(1.3)$ & $0(0.0)$ & - & $>0.99$ & & \\
\hline Other & & $1(0.7)$ & $0(0.0)$ & - & $>0.99$ & & \\
\hline Multifocal infection, $n(\%)$ & 188 & $14(9.3)$ & $5(13.2)$ & - & 0.540 & & \\
\hline Shock, $n(\%)$ & 185 & $48(31.8)$ & $31(81.6)$ & $8.20[3.40-20.00]$ & $<0.001$ & $8.13[3.26-20.20]$ & $<0.001$ \\
\hline
\end{tabular}

HIV human immunodeficiency virus, NSAID non-steroidal anti-inflammatory drugs

Univariable and multivariable analysis by Cox proportional hazards model. Multivariable analysis for 179 patients (10 excluded for missing data on one of the variables)

a,b Hazard ratios and $p$ values from the comparison of survivors and non-survivors by univariate cox regression analysis for survival censored at 60 days

$c, d$ Adjusted hazard ratios and $p$ values from a multivariate cox model for survival censored at 60 days. Only patients from the pre- and post-implementation periods were included in the analysis. Variables included in the model were all variables available upon admission associated with mortality in the univariate model with a $p$ value inferior or equal to 0.05 . Corticosteroid treatment was included as part of immunodeficiency. Included variables with a $p$ value $>0.1$ (chronic kidney disease) in the multivariate model were excluded from the final model

the impact of age and pre-existing comorbidities on mortality, rather than that of the first $48 \mathrm{~h}$ of management on which our bundle mainly focused. Finally, the fact that antibiotics administered before admission were a protective factor for hospital survival in the multivariable model is remarkable and may have limited our ability to demonstrate a benefit of antibiotics administered within $24 \mathrm{~h}$ of hospital admission.

This work has several limitations, the first of which being its monocentric retrospective design, limiting the generalization of its results. Second, time to surgery was measured in days, not in hours, which could have 


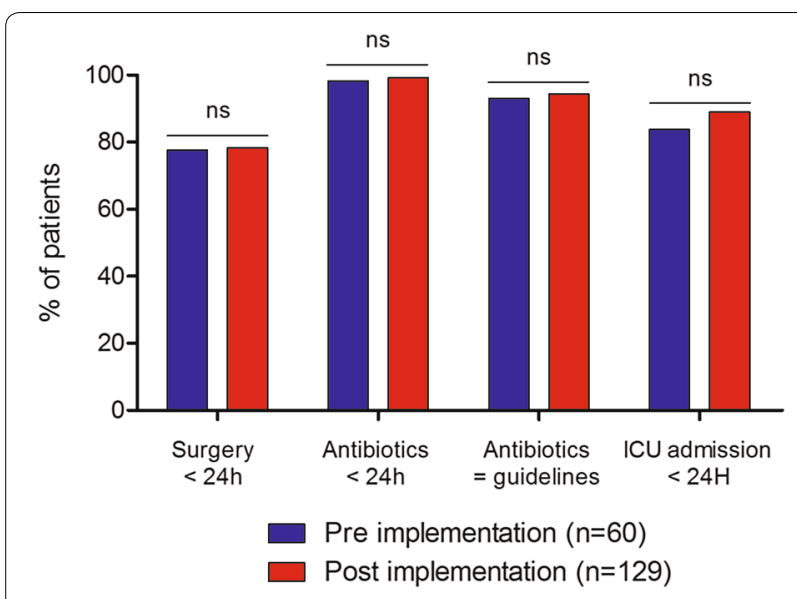

Fig. 3 Impact of the implementation of a multimodal bundle of care on pre-defined patient management outcomes. Patients from the pre- (blue histograms) and post-implementation (red histograms) periods are compared. None of the comparisons yielded statistically different results by Fisher's exact test or the Chi-squared test according to sample size

undermined our ability to demonstrate its impact on outcome. As patients were identified with two different methods according to the period of inclusion (electronic records or prospective database) and because 14 patients with important missing data were excluded without imputation, we cannot exclude a selection bias. Antibiotic treatment was not evaluated for adequacy to documentation, duration, de-escalation and side effects. We could not obtain time to administration in hours, due to the retrospective design of the study. Finally, elements not included in our bundle and that were not evaluated could have impacted outcome, such as use of anti-toxinic antibiotics or negative-pressure wound therapy [26].

Our study also has several strengths, the first of which is a well-defined diagnosis as a main inclusion criterion. Indeed, an important part of the literature on NSTIs has included patients based on an electronic record diagnosis of NSTIs, a major selection bias for a disease with a challenging diagnosis [23]. We only included surgically proven NSTIs, and in spite of this restrictive definition, the second strength of this cohort is its large size. Finally, we applied a rigorous methodology to this before-after study, defining key management endpoints a priori, and choosing a strong outcome measure (i.e., hospital mortality).

Interestingly, during the conduction of this project, the only other report, to our knowledge, of a multidisciplinary bundle of care for NSTIs was published. Although focusing on ICU patients, this much smaller series found a very similar benefit on mortality to their bundle $(40 \%$ vs $15 \%$ after implementation, compared to
$30 \%$ vs $15 \%$ in our work), highlighting this approach's potential interest [27]. By contrast to the hospital that conducted this research, our center has been a referral center for NSTIs for several decades. Standard of care before bundle implementation likely rendered patient management homogeneous, making it difficult to show a statistically significant difference on a solid outcome like mortality. Nevertheless, our work confirms the feasibility of standardizing multidisciplinary care for NSTIs on a larger scale.

\section{Conclusions}

Although this retrospective work from a center with preexisting expertise could not find a benefit on mortality to the implementation of a multidisciplinary care bundle for NSTIs after adjustment for confounding factors, it highlights its feasibility and potential interests, such as increasing patient recruitment. Every practitioner confronted with this rare, severe and often littleknown condition, especially at the time of triage, could benefit from implementing a multidisciplinary bundle appointing specialists involved in NSTI management to optimize and speed up treatment. Future work, ideally prospective, could benefit from and abound in favor of the approach we describe.

\section{Supplementary information}

Supplementary information accompanies this paper at https://doi. org/10.1186/s13613-019-0598-4.

Additional file 1. The study was approved by the Comité de Protection des Personnes Ile-de-France V on March 8th 2018 (reference \# 16165).

Additional file 2: Appendix S1. Details on the necrotizing soft-tissue infection task force and on bundle items.

\section{Abbreviations}

aHR: adjusted hazard ratio; Cl: confidence interval; HIV: human immunodeficiency virus; HR: hazard ratio; ICU: intensive care unit; IQR: interquartile range; NSAID: non-steroidal anti-inflammatory drug; NSTI: necrotizing soft tissue infection.

\section{Acknowledgements}

Tomas Urbina thanks Yann Nguyen for his helpful advice on data management and statistical analysis.

\section{Authors' contributions}

All authors were involved in study conception and design. TU and NdP collected data, performed statistical analyses and wrote the original draft. All authors were involved in interpreting data and reviewing the final manuscript. All authors read and approved the final manuscript.

Funding

This work did not receive any funding.

\section{Availability of data and materials}

The datasets used and/or analyzed during the current study are available from the corresponding author on reasonable request. 


\section{Consent for publication \\ Not applicable.}

\section{Competing interests}

The authors declare that they have no competing interests.

\section{Author details}

1 Service de Réanimation Médicale, Hôpitaux Universitaires Henri Mondor, Assistance Publique-Hôpitaux de Paris (AP-HP), Créteil, France. ${ }^{2}$ Service de Dermatologie, Hôpitaux Universitaires Henri Mondor, Assistance PubliqueHôpitaux de Paris (AP-HP), Créteil, France. ${ }^{3}$ Université Paris-Est Créteil Val de Marne (UPEC), Créteil, France. ${ }^{4}$ Epidemiology in Dermatology and Evaluation of Therapeutics (EpiDermE), EA7379, Université Paris Est-Créteil, DHU VIC Créteil, France. ${ }^{5}$ Service de Chirurgie Plastique et Reconstructrice, Hôpitaux Universitaires Henri Mondor, Assistance Publique-Hôpitaux de Paris (AP-HP), Créteil, France. ${ }^{6}$ Service d'Anesthésie et des Réanimations Chirurgicales, Hôpitaux Universitaires Henri Mondor, Assistance Publique-Hôpitaux de Paris (APHP), Créteil, France. ${ }^{7}$ Unité Transversale de Traitement des Infections, Hôpitaux Universitaires Henri Mondor, Assistance Publique-Hôpitaux de Paris (AP-HP), Créteil, France. ${ }^{8}$ Laboratoire de Bactériologie-Hygiène, Hôpitaux Universitaires Henri Mondor, Assistance Publique-Hôpitaux de Paris (AP-HP), Créteil, France. ${ }^{9}$ Équipe EA 7380 Dynamyc, Unité de Formation et de Recherche (UFR) de Médecine-Site Créteil, Université Paris-Est Créteil Val-de-Marne, Créteil, France. ${ }^{10}$ Groupe de Recherche Clinique CARMAS, Université Paris Est-Créteil, Créteil, France.

Received: 9 August 2019 Accepted: 15 October 2019 Published online: 24 October 2019

\section{References}

1. Stevens DL, Bryant AE. Necrotizing soft-tissue infections. N Engl J Med. 2017;377(23):2253-65.

2. Arif $N$, Yousfi S, Vinnard C. Deaths from necrotizing fasciitis in the United States, 2003-2013. Epidemiol Infect. 2016;144(06):1338-44.

3. Gawaziuk JP, Strazar R, Cristall N, Logsetty S. Factors predicting health-related quality of life following necrotizing fasciitis. J Plast Reconstr Aesthet Surg. 2018;71(6):857-62.

4. Soltani AM, Best MJ, Francis CS, Allan BJ, Askari M, Panthaki ZJ. Trends in the incidence and treatment of necrotizing soft tissue infections: an analysis of the National Hospital Discharge Survey. J Burn Care Res. 2014;35:449-54.

5. Goh T, Goh LG, Ang CH, Wong CH. Early diagnosis of necrotizing fasciitis: early diagnosis of necrotizing fasciitis. Br J Surg. 2014;101(1):e119-25.

6. Boyer A, Vargas F, Coste F, Saubusse E, Castaing Y, Gbikpi-Benissan G, et al. Influence of surgical treatment timing on mortality from necrotizing soft tissue infections requiring intensive care management. Intensive Care Med. 2009;35(5):847-53.

7. de Prost N, Sbidian E, Chosidow O, Brun-Buisson C, Amathieu R. Management of necrotizing soft tissue infections in the intensive care unit: results of an international survey. Intensive Care Med. 2015;41(8):1506-8.

8. Audureau E, Hua C, de Prost N, Hemery F, Decousser JW, Bosc R, et al. Mortality of necrotizing fasciitis: relative influence of individual and hospitallevel factors, a nationwide multilevel study, France, 2007-12. Br J Dermatol. 2017;177(6):1575-82.

9. Hua C, Bosc R, Sbidian E, De Prost N, Hughes C, Jabre P, et al. Interventions for necrotizing soft tissue infections in adults. Cochrane Database Syst Rev. 2018:5:CD011680

10. Roussel A, Castier Y, Nuzzo A, Pellenc Q, Sibert A, Panis Y, et al. Revascularization of acute mesenteric ischemia after creation of a dedicated multidisciplinary center. J Vasc Surg. 2015;62(5):1251-6.
11. Seymour CW, Gesten F, Prescott HC, Friedrich ME, Iwashyna TJ, Phillips GS, et al. Time to treatment and mortality during mandated emergency care for sepsis. N Engl J Med. 2017;376(23):2235-44.

12. MacKenzie EJ, Rivara FP, Jurkovich GJ, Nathens AB, Frey KP, Egleston BL, et al. A national evaluation of the effect of trauma-center care on mortality. $\mathrm{N}$ Engl J Med. 2006;354(4):366-78.

13. Stroke Unit Trialists' Collaboration. Organised inpatient (stroke unit) care for stroke. In: The Cochrane Collaboration, editor. Cochrane database of systematic reviews. Chichester: Wiley; 2007.

14. Stevens DL, Bisno AL, Chambers HF, Dellinger EP, Goldstein EJC, Gorbach SL, et al. Practice guidelines for the diagnosis and management of skin and soft tissue infections: 2014 update by the infectious diseases society of America. Clin Infect Dis. 2014;59(2):e10-52.

15. Spilf et SFD. Conférence de consensus Erysipèle et fasciite nécrosante: prise en charge. Med Mal Inf. 2000;30:241-5.

16. Bernal NP, Latenser BA, Born JM, Liao J. Trends in 393 necrotizing acute soft tissue infection patients 2000-2008. Burns J Int Soc Burn Inj. 2012;38(2):252-60.

17. Oud L, Watkins P. Contemporary trends of the epidemiology, clinical characteristics, and resource utilization of necrotizing fasciitis in texas: a population-based cohort study. Crit Care Res Pract. 2015;2015:1-9.

18. Psoinos CM, Flahive JM, Shaw JJ, Li Y, Ng SC, Tseng JF, et al. Contemporary trends in necrotizing soft-tissue infections in the United States. Surgery. 2013;153(6):819-27.

19. Van Stigt SFL, de Vries J, Bijker JB, Mollen RMHG, Hekma EJ, Lemson SM, et al. Review of 58 patients with necrotizing fasciitis in the Netherlands. World J Emerg Surg. 2016;11:1.

20. Faraklas I, Yang D, Eggerstedt M, Zhai Y, Liebel P, Graves G, et al. A multicenter review of care patterns and outcomes in necrotizing soft tissue infections. Surg Infect. 2016;17(6):773-8.

21. Zhao J-C, Zhang B-R, Shi K, Zhang X, Xie C-H, Wang J, et al. Necrotizing soft tissue infection: clinical characteristics and outcomes at a reconstructive center in Jilin Province. BMC Infect Dis. 2017;17(1):792.

22. Holena DN, Mills AM, Carr BG, Wirtalla C, Sarani B, Kim PK, et al. Transfer status: a risk factor for mortality in patients with necrotizing fasciitis. Surgery. 2011;150(3):363-70.

23. Ingraham AM, Jung HS, Liepert AE, Warner-Hillard C, Greenberg CC, Scarborough JE. Effect of transfer status on outcomes for necrotizing soft tissue infections. J Surg Res. 2017;220:372-8.

24. Hua C, Sbidian E, Hemery F, Decousser JW, Bosc R, Amathieu R, et al. Prognostic factors in necrotizing soft-tissue infections (NSTI): a cohort study. J Am Acad Dermatol. 2015;73(6):1006-12.

25. Bucca K, Spencer R, Orford N, Cattigan C, Athan E, McDonald A. Early diagnosis and treatment of necrotizing fasciitis can improve survival: an observational intensive care unit cohort study: early diagnosis and treatment of NF. ANZ J Surg. 2013;83(5):365-70.

26. Saeed K, Esposito S, Gould I, Ascione T, Bassetti M, Bonnet E, et al. Hot topics in necrotising skin and soft tissue infections. Int J Antimicrob Agents. 2018:52:1-10.

27. Gatti M, Gasparini LE, Laratta M, Sigurtà A, Rossi A, Brioschi P, et al. Intensive multidisciplinary management in critical care patients affected by severe necrotizing soft tissue infections: a cooperative method to improve the efficacy of treatment. Eur J Clin Microbiol Infect Dis. 2019;38:1153-62.

\section{Publisher's Note}

Springer Nature remains neutral with regard to jurisdictional claims in published maps and institutional affiliations. 